\title{
Planar Antenna Array on LTCC and Rogers substrates for $5 G$ applications
}

\author{
Rui Zhang , Xiaoliang Sun , José-Manuel Fernández-González ,Manuel Sierra-Pérez
}

\begin{abstract}
The next generation of wireless communications is the horizon, and the telecoms world is waiting to see exactly at which frequencies 5G will be implemented. Despite some unknowns and technological challenges, it is increasingly becoming clear that the millimeter-wave will be a key enabler for $5 \mathrm{G}$ by allocating more bandwidth to deliver faster, higher-quality video, and multimedia content and services. The principal advantage of use of millimeter wave bands is a large amounts of contiguous spectrum can be used, which allows the delivery of high data rates, but the technology is not so mature as in microwave frequencies. This paper presents array antennas using different materials: low-temperature cofired ceramics (LTCC) and Rogers substrates of a single radiating element, $1 \times 1$ and $2 \times 2$ array. The $2 \times 2$ element array antenna achieves a $-10 \mathrm{~dB}$ bandwidth from 58 to $62 \mathrm{GHz}$ with a peak gain of $12.2 \mathrm{dBi}$ at $60 \mathrm{GHz}$.
\end{abstract}

Keywords- aperture-coupled patch antenna, LTCC, Multi-gigabit communication; $60 \mathrm{GHz}$ band; $5 \mathrm{G}$ application.

\section{INTRODUCTION}

Nowadays, consumer device bandwidth requirements are increasing at rapid rates. Historical bands below $3 \mathrm{GHz}$ become crowded, which pushes the development of 5G. At millimeterwave bands, atmospheric propagation effects can be ignored within around $1 \mathrm{~km}$ and antenna size is small enough to be used both in user terminal equipment and base stations [1]. The large $7-9 \mathrm{GHz}$ of unlicensed spectrum band at $60 \mathrm{GHz}$ attracts us with multi-gigabit per second data rates [2]. However, the signal propagation path loss and high packaging integration loss are normally higher than $20 \mathrm{~dB}$ [3], which challenges the design of high-gain antennas.

Low-temperature cofired ceramic (LTCC) has been regarded as a promising technology for its flexibility in realizing a number of layers and its highly compact vertical integration [4]. Since the conductor layers in LTCC materials are screen-printed, aperture coupled microstrip slot antennas [5] are a good choice to reduce back radiation, which is an inconvenience in mobile communication. At $60 \mathrm{GHz}$ band, microstrip slot antenna is very small and lightweight.

In this paper, we designed some prototypes on Rogers substrates to compare with the results of LTCC at $60 \mathrm{GHz}$ for $5 \mathrm{G}$ application. Six of them with $1 \times 1,1 \times 2$ and $2 \times 2$ patches are realized on LTCC. Obtained bandwidths are up to $8 \%$ and gains up to $12.2 \mathrm{dBi}$. Another six are fabricated on substrate, achieving a wide bandwidth of up to $5.2 \%$, with a superior gain of $11 \mathrm{dBi}$.

\section{Antenna DESIGN}

\section{A. Single Patch Antenna}

Fig.1(a) presents the structure of the proposed aperture-coupled patch antenna using LTCC. The top substrate is a $0.336 \mathrm{~mm}-$ thick layer of DuPont $9 \mathrm{~V} 7 \mathrm{k}$, the bottom substrate is a $0.112 \mathrm{~mm}$ thick layer of the same material. The relative permittivity $\varepsilon_{R}$ of multilayer LTCC substrate is 7.1. Fig.1(b) presents the proposed aperture-coupled patch antenna on Rogers substrates. The top substrate is a $0.127 \mathrm{~mm}$-Rogers 5880 , whose relative permittivity $\varepsilon_{\mathrm{R}}$ is 2.2 , followed by a $0.050 \mathrm{~mm}$-thick Rogers 2929 , whose relative permittivity $\varepsilon_{\mathrm{R}}$ is 2.94 . The stacked layers below the Rogers 2929 are respectively a 0.018 mm-thick groundplane with an aperture of $0.4 \mathrm{~mm} \times 0.65 \mathrm{~mm}$ and a 0.254 mm-thick Rogers 6010 , whose relative permittivity $\left(\varepsilon_{\mathrm{R}}\right)$ is 10.2 .

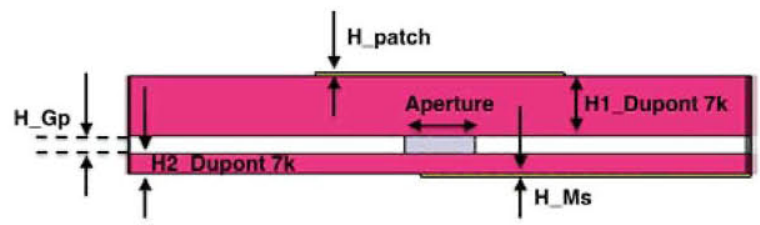

Fig.1. (a) Stack-up of Aperture-coupled patch using LTCC.

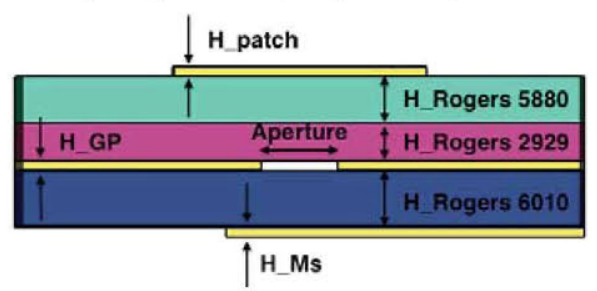

Fig.1. (b) Stack-up of Aperture-coupled patch using Rogers substrate.

\section{B. Multi-Element Antenna Array}

To achieve greater directivity and gain, $1 \times 2$ and $2 \times 2$ array antennas of the previous basic design are proposed. The sizes of each element remain the same. The quarter-wave transformer strip line is used to maintain a $50 \Omega$ impedance.

However, the discontinuity of the power divider increases the back radiation. To reduce back lobe of radiation pattern, Fig. 2 presents the structure of $1 \times 2$ array antenna. This junction angle was optimized to distribute the equal power to each radiating element. To reduce the mutual coupling between radiating elements, the optimized value of the inter-element spacing is chosen to be $0.6 \lambda_{0}$.

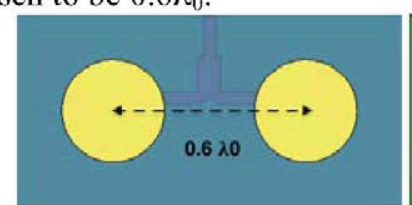

(a) Top view.

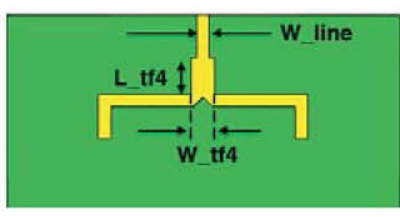

(b) Bottom view.
Fig.2. Structure of $1 \times 2$ array antenna. 
Fig. 3 presents the structure of $2 \times 2$ array antenna. The upper two patches are given 180-degrees phase rotation respect to the pair below instead of using an equal feeding phase approach.

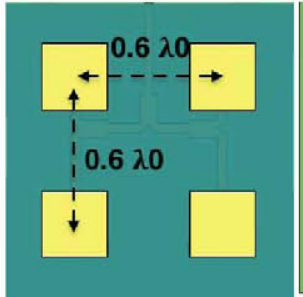

(a) Top view.

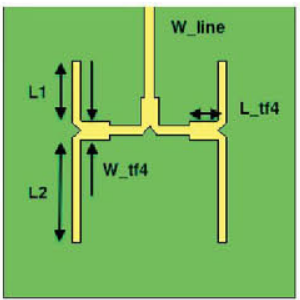

(b) Bottom view.
Fig.3. Feeding network of $2 \times 2$ array antenna.

Table I presents the optimized dimensions of the proposed array using CST MWS.

\begin{tabular}{|l|l|l|l|l|l|}
\hline Param(mm) & LTCC & Rogers & Param(mm) & LTCC & Rogers \\
\hline W_line & 0.12 & 0.19 & L_tf4 & 0.36 & 0.56 \\
\hline L1 & 0.89 & 1.25 & Aperture & $0.4 \times 0.65$ & $0.4 \times 0.65$ \\
\hline L2 & 2.06 & 2.15 & L_patch & 0.64 & 1.34 \\
\hline W_tf4 & 0.33 & 0.36 & R_patch & 1.33 & 0.79 \\
\hline
\end{tabular}

TABLE I.

OPTIMIZED ANTENNA DIMENSIONS.

\section{COMPARISON BETWEEN LTCC AND ROGERS}

\section{A. Gain}

Table II presents a summary of the gain results that have been obtained using Rogers substrates and LTCC approaches. The $2 \times 2$ array antenna with circular patches using LTCC achieves the highest gain of $12.2 \mathrm{~dB}$ at $60 \mathrm{GHz}$.

\begin{tabular}{|c|c|c|c|c|c|c|c|}
\hline \multicolumn{2}{|c}{ Gain (dB) } & \multicolumn{3}{c|}{ Circular } & \multicolumn{3}{c|}{ Square } \\
\cline { 2 - 8 } & & $\mathbf{1} \boldsymbol{x} \mathbf{1}$ & $\mathbf{1} \boldsymbol{x} \mathbf{2}$ & $\mathbf{2 x} 2$ & $\mathbf{1} \boldsymbol{x} \mathbf{1}$ & $\mathbf{1} \boldsymbol{x} \mathbf{2}$ & $\mathbf{2} \boldsymbol{x} \mathbf{2}$ \\
\hline \multirow{3}{*}{ LTCC } & $\mathrm{f}-59 \mathrm{GHz}$ & 6.22 & 7.68 & 11.8 & 5.45 & 7.92 & 11.2 \\
\cline { 2 - 8 } & $\mathrm{f}=60 \mathrm{GHz}$ & 7.39 & 8.69 & 12.2 & 5.41 & 7.88 & 11.5 \\
\cline { 2 - 8 } & $\mathrm{f}=61 \mathrm{GHz}$ & 8.19 & 9.43 & 12.5 & 5.36 & 7.82 & 11.6 \\
\hline \multirow{3}{*}{ Rogers } & $\mathrm{f}=59 \mathrm{GHz}$ & 6.56 & 7.69 & 11 & 6.55 & 7.8 & 10.8 \\
\cline { 2 - 8 } & $\mathrm{f}=60 \mathrm{GHz}$ & 6.54 & 7.36 & 11.1 & 6.49 & 7.6 & 10.8 \\
\cline { 2 - 8 } & $\mathrm{f}=61 \mathrm{GHz}$ & 6.43 & 6.89 & 11 & 6.34 & 7.19 & 10.6 \\
\hline
\end{tabular}

TABLE II. GAIN OF ROGERS SUBSTRATES AND LTCC.

\section{B. Beamwidth}

Table III presents a summary of the beamwidth results that have been obtained using Rogers substrates and LTCC approaches at $\varphi=0^{\circ}$ and $\varphi=90^{\circ}$. The $2 \times 2$ element array using LTCC achieves narrow beamwidth.

\begin{tabular}{|c|c|c|c|c|c|c|c|}
\hline \multicolumn{2}{|c|}{ Beamwidth $\left({ }^{\circ}\right)$} & \multicolumn{3}{|c|}{ Circular } & \multicolumn{3}{c|}{ Square } \\
\cline { 3 - 8 } & & $\mathbf{1} \boldsymbol{x}$ & $\mathbf{1} \boldsymbol{x} \mathbf{2}$ & $\mathbf{2 \times 2}$ & $\mathbf{1} \boldsymbol{1}$ & $\mathbf{1} \mathbf{2}$ & $\mathbf{2 \times 2}$ \\
\hline \multirow{2}{*}{ LTCC } & $\varphi=0^{\circ}$ & 65.4 & 36.8 & 37.1 & 84.7 & 39 & 34.1 \\
\cline { 2 - 8 } & $\varphi=90^{\circ}$ & 63.0 & 59.6 & 37.3 & 109.4 & 105.7 & 42.3 \\
\hline \multirow{2}{*}{ Rogers } & $\varphi=0^{\circ}$ & 81.2 & 48.4 & 45.8 & 81.0 & 48.7 & 46.1 \\
\cline { 2 - 8 } & $\varphi=90^{\circ}$ & 86.8 & 81.7 & 43.8 & 89.4 & 80.7 & 43.5 \\
\hline
\end{tabular}

TABLE III. COMPARISON BEAMWIDTH AT 60 GHZ.

\section{S-parameter}

Fig. 4 presents the $S_{11}$ curves of $2 \times 2$ antenna arrays of circular or square patches using Rogers substrates and LTCC.

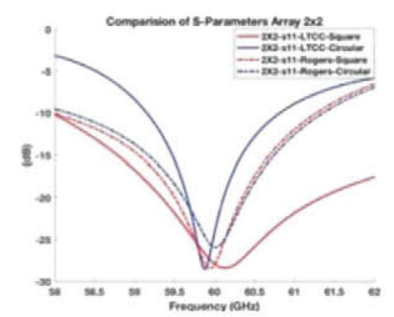

Fig.4. $2 \times 2$ Array Comparison of $\mathrm{S}_{11}$.

\section{Radiation Pattern of the best option}

Fig.5. presents the radiation pattern of the $2 \times 2$ LTCC array antenna with the highest gain.

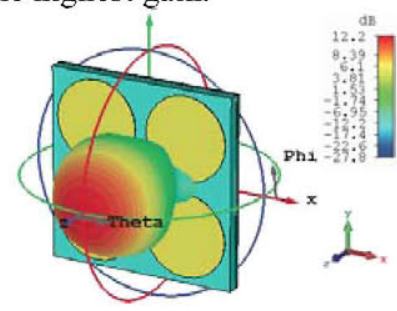

Fig.5. Radiation Pattern of the $2 \times 2$ LTCC array antenna.

\section{Conclusion}

Planar antenna arrays are presented at $60 \mathrm{GHz}$ with two different materials: LTCC and Rogers substrates. From the results of simulation, we can find that LTCC has a better performance at $60 \mathrm{GHz}$ band in comparison to Rogers substrates in the aspects of S-parameter, bandwidth, losses, crosspolar, gain and directivity. The $2 \times 2$ element array with circular patch using LTCC can achieve a maximum gain of $12.2 \mathrm{~dB}$ at $60 \mathrm{GHz}$. Prototype measurement results will be presented in ISAP 2017.

\section{ACKNOWLEDGMENT}

The simulations done in this work have been carried out using CST Studio Suite under a cooperation agreement between Computer Simulation Technology and Universidad Politécnica de Madrid. This work has been supported by the project ENABLING5G, "Enabling Innovative Radio Technologies for 5G networks" (TEC2014-55735-C3-1-R) of the Spanish Research and Development National Program and by the project S2013/ICE-3000 SPADERADAR-CM of the Madrid Region Government.

\section{REFERENCES}

[1] Li R L, Dejean G, Maeng M, et al. Design of compact stacked-patch antennas in LTCC multilayer packaging modules for wireless applications[J]. IEEE Transactions on Advanced Packaging, 2004, 27(4):581-589.

[2] Pan H K, Ruberto M, Horine B D, et al. MM-WAVE PHASED ARRAY ANTENNA AND SYSTEM INTEGRATION ON SEMI-FLEX PACKAGING: IEEE, WO/2012/125774[P]. 2012.

[3] Chin K S, Chang H T, Liu J A, et al. 28-GHz patch antenna arrays with PCB and LTCC substrates[C]// Cross Strait Quad-Regional Radio Science and Wireless Technology Conference. IEEE, 2011:355-358.

[4] Panther A, Petosa A, Stubbs M G, et al. A wideband array of stacked patch antennas using embedded air cavities in LTCC[J]. Microwave \& Wireless Components Letters IEEE, 2006, 15(12):916-918.

[5] Aijaz Z, Shrivastava S C. An Introduction of Aperture Coupled Microstrip Slot Antenna[J]. International Journal of Engineering Science \& Technology, 2010,2(1). 